\title{
Fast and accurate modeling of waveguide grating couplers. II. Three-dimensional vectorial case
}

\author{
P. G. Dinesen \\ Department of Optics and Fluid Dynamics, Risø National Laboratory, OFD-128, P.O. Box 49, DK-400o Roskilde, \\ Denmark
}

J. S. Hesthaven

Division of Applied Mathematics, Brown University, Box F, Providence, Rhode Island 02912

Received January 10, 2001; revised manuscript received March 30, 2001; accepted March 27, 2001

\begin{abstract}
A boundary variation method for the fast and accurate modeling of three-dimensional waveguide grating couplers is presented. The algorithm is verified by detailed comparisons with the results of a rigorous spectral collocation method, showing excellent agreement. Examples of the modeling of large waveguide grating couplers are given to illustrate the applicability and versatility of the method. () 2001 Optical Society of America

OCIS codes: $050.1960,000.4430$.
\end{abstract}

\section{INTRODUCTION}

The growing awareness of the possibilities that diffraction-based optical components offer as an enabling technology in the realization of miniaturized optical systems continues to stretch the capabilities of existing numerical methods in an attempt to analyze and design such novel optical components. Such numerical modeling and design efforts are made difficult by the very nature of the diffraction-based optics, which is characterized by complex geometric shapes and extends hundreds if not thousands of wavelengths, although often with important effects caused by its finite size. At the heart of the problem, however, is the requirement that the full vectorial wave nature of fields be accounted for over long times and with high accuracy to permit correct modeling of the phase-sensitive components.

The combination of these factors makes the full threedimensional modeling of diffractive optical elements (DOEs) a significant computational challenge and essentially rules out the use of standard computational techniques such as finite-difference time-domain and finiteelement methods, because of excessive computational expense, and methods that require periodicity, such as rigorous coupled-wave methods. We previously advocated the use of pseudospectral multidomain methods ${ }^{1,2}$ as a feasible approach and demonstrated that these methods offer the ability to model general optical components of a realistic size to the required accuracy. For fixed design studies these methods remain the best and perhaps the only way to model a general large-scale optical component.

However, with the growing appreciation of the properties of DOEs it becomes increasingly important to attempt the development of design tools as opposed to analysis tools, for which the aforementioned pseudospec- tral multidomain methods are eminently useful. Unfortunately, even with the highly efficient spatial representation of the capabilities of these methods, the required computational resources are still quite substantial, and systematic design studies are not feasible now. For such studies one needs fast solvers to permit multiple parameter variations and, ultimately, the development of automated inverse optimal design tools.

It is with this ultimate goal in mind that we have set out to develop a new class of tool, less general in nature than the pseudospectral multidomain methods but specifically designed to permit fast and accurate modeling of waveguide grating couplers used for controlling the coupling between a thin-film optical waveguide and free space. We have discussed in detail the formulation of a two-dimensional boundary variation method that is suitable for the analysis of waveguide grating couplers. ${ }^{3}$ At the heart of the method is the observation, originally made by Bruno and Reitich, ${ }^{4}$ that the field that has been diffracted at a nonsmooth surface can be found by analytic continuation of the field refracted at the corresponding smooth interface. Hence the computation of the solution reduces to that of a summation of a Taylor series, the coefficients of which can be found by a recurrence. Although this method, in the case of a waveguide coupler, is only approximate, leaving out the effect of power loss in the guided wave as a result of radiation, we demonstrated in Ref. 3 that in the two-dimensional case the boundary variation approach yields results for finite-sized realistic configurations of a high fidelity at a fraction of the computational cost required for direct numerical simulation.

Here we extend the boundary variation methods to the full three-dimensional waveguide grating problem required for modeling general two-dimensional surfacerelief gratings. The scalar wave equation is not sufficient 
to govern three-dimensional wave propagation, which requires that the vector wave equation be considered for all three components of either the electric or the magnetic field. As we shall demonstrate, the conclusions on accuracy and computational efficiency for the two-dimensional case carry over to the general three-dimensional grating, confirming that the boundary variation method proposed here offers a fast and accurate forward solver that is suitable for integration into an optimal design loop.

What remains of the paper is organized as follows: In Section 2 the basic waveguide grating coupler is described; Section 3 is devoted to the development of the three-dimensional boundary variation method suitable for such problems. The accuracy and overall performance of the proposed method are discussed in Section 4 by thorough comparisons with direct simulation results obtained from a general three-dimensional pseudospectral scheme. This sets the stage for Section 5, in which we discuss the modeling of nontrivial waveguide grating couplers. Section 6 contains a few concluding remarks and an outlook for continued efforts along the lines described here.

\section{WAVEGUIDE GRATING COUPLERS WITH TWO-DIMENSIONAL SURFACE RELIEFS}

The basis for the waveguide grating couplers discussed in this paper is a thin film with a core layer sandwiched between a semi-infinite lower cladding layer and an upper layer with a finite thickness, as illustrated schematically in Fig. 1. The central idea is to modulate the upper cladding layer in such a way that controlled coupling of energy from the waveguide into free space is achieved.

In the two-dimensional case, in which the surface relief is one dimensional, focusing of the free-space radiation is achieved by chirping of the sinusoidal surface relief such that the grating period becomes gradually smaller in the direction of propagation of the incident guided wave. In the three-dimensional case, for which the surface relief is two-dimensional, focusing must be achieved in both transverse directions. This can be accomplished by chirping in the direction of propagation of the incident wave. In the transverse direction perpendicular to this, one can realize focusing by shaping the surface relief as

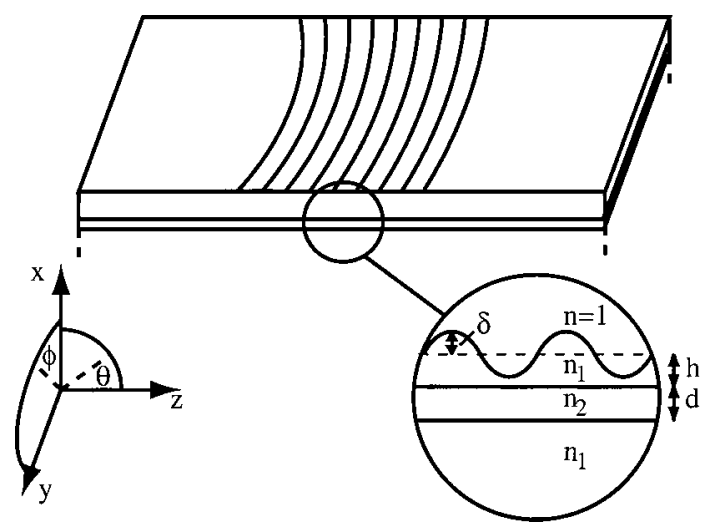

Fig. 1. Waveguide grating coupler with two-dimensional surface relief for controlled coupling between a thin-film guided wave and free-space radiation. concentric circles, the center of which is shifted some distance from the center of the whole relief. This shift in turn determines the focal length of the surface-relief coupler in that direction.

In what follows, we denote by $\mathbf{E}^{+}$the diffracted field radiated into free space above the surface relief, whereas the field radiating into the waveguide structure is denoted $\mathbf{E}^{-}$. In computing the upward-diffracted field, we neglect the contributions from the partial reflection of the downward-radiated field at the lower-lying interfaces between the cladding and the core layers and the core and the lower cladding layers. Because the index difference is quite small, this assumption is reasonable, and, as we shall show, our validation of the proposed scheme bears no evidence of these contributions.

Often, it is desired to have the -1st diffraction order located at $\theta=\pi / 2, \phi=0$ such that the direction of propagation of the radiation is perpendicular to the surface relief and hence to the waveguide. To achieve $\phi=0$ requires that the surface relief be symmetric with respect to the $y=0$ plane, and to achieve a perpendicular output requires that the Bragg condition

$$
0=n_{\text {eff }}-(\lambda / \Lambda)
$$

be obeyed. $n_{\text {eff }}$ is the effective propagation constant of the guided wave, $\lambda$ is the free-space wavelength of the monochromatic wave, and $\Lambda$ is the period of the unchirped grating. Hence, normalized grating period $\Lambda / \lambda$ is the inverse of the effective index of the fundamental guided mode in the thin-film waveguide.

In the numerical examples that we give below, we use values for the basic waveguide that are representative of optical materials such as glass and polymers: We consider a waveguide structure consisting of a core layer with refractive index $n_{2}=1.45$ and thickness $d=0.8 \lambda$ sandwiched between two cladding layers of refractive index $n_{1}=1.4$. The top cladding layer has a finite thickness $h=1$, and above this layer is air with $n=1$. There is no confinement of the guided wave in the $y$ direction. For the fundamental TE mode this geometry yields an effective propagation constant, $n_{\text {eff }}=1.4213$.

\section{BOUNDARY VARIATIONS FOR A WAVEGUIDE GRATING COUPLER}

In Ref. 5 it is shown that the diffracted fields, $\mathbf{E}(x, y, z ; \delta)$ and $\mathbf{H}(x, y, z ; \delta)$, are analytic with respect to variations in grating amplitude $\delta$ (cf. Fig. 1). The implication of this result is that we can recover the solutions to complicated diffraction problems by the analytic continuation of solutions to simple scattering problems for which analytic solutions may be known. This is the fundamental result that underlies the boundary variation methods that we shall discuss further below.

Let us in what follows outline the numerical algorithm, based on the above result, that we subsequently use to model large three-dimensional waveguide grating couplers.

As was mentioned above, we need, unlike in the scalar two-dimensional case, to consider the vectorial Helmholtz equation to model the three-dimensional scattering problem. Hence the electric field must satisfy 


$$
\Delta \mathbf{E}+k^{2} \mathbf{E}=0,
$$

from which the magnetic field can be recovered as

$$
\mathbf{H}=\frac{1}{i \omega \mu_{0}}(\nabla \times \mathbf{E}) .
$$

Under the assumption of $(z, y)$ periodicity, the diffracted electric field can be expanded in a Rayleigh series:

$$
\mathbf{E}^{ \pm}=\sum_{r=-\infty}^{\infty} \sum_{s=-\infty}^{\infty} \mathbf{B}_{r, s}^{ \pm} \exp \left( \pm i \alpha_{r, s}^{ \pm} x+i \gamma_{s} y+i \beta_{r} z\right)
$$

where $\mathbf{E}^{+}$denotes the portion of the diffracted field that is radiated upward and $\mathbf{E}^{-}$denotes the portion of the diffracted field that is radiated downward into the waveguide structure. $\beta_{0}$ and $\gamma_{0}$ are the $z$ and $y$ components, respectively, of the incident wave vector. Further,

$$
\begin{aligned}
\beta_{r} & =\beta_{0}+r K_{z}, \\
\gamma_{s} & =\gamma_{0}+s K_{y}, \\
\left(\alpha_{r, s}^{ \pm}\right)^{2}+\beta_{r}^{2}+\gamma_{s}^{2} & =\left(k^{ \pm}\right)^{2} .
\end{aligned}
$$

Here $k^{ \pm}$are the wave numbers above and below the boundary, respectively, and $K_{z}$ and $K_{y}$ represent the smallest grating vectors that we wish to resolve. For a biperiodic grating, these are simply the grating wave numbers, whereas for a finite, aperiodic grating, these grating vectors are given by $K_{z}=2 \pi / L_{z}$ and $K_{y}$ $=2 \pi / L_{y}$, where $L_{z}$ and $L_{y}$ are the sizes of the computational domain in the $z$ and the $y$ directions, respectively.

Let us now introduce a two-dimensional surface relief. We describe it by amplitude $\delta$ times function $f(z, y)$ such that the actual surface relief lies on the surface given by

$$
x(z, y)=f_{\delta}(z, y)=\delta f(z, y) .
$$

Because of the analyticity of the diffracted field in $\delta$, the Rayleigh coefficients in expansion (2) may be expressed by a Taylor series expansion:

$$
\mathbf{B}_{r, s}^{ \pm}=\sum_{p=0}^{\infty} \mathbf{d}_{p,(r, s)}^{ \pm} \delta^{p},
$$

where $\mathbf{d}_{p,(r, s)}^{ \pm}=\left(d_{p,(r, s)}^{1, \pm}, d_{p,(r, s)}^{2, \pm}, d_{p,(r, s)}^{3, \pm}\right)$ are the Taylor expansion coefficients. The superscripts 1,2 , and 3 refer to the $x, y$, and $z$ components, respectively, of $\mathbf{d}$. This notation is used throughout what follows. The central result in the realization of the boundary variation method is that these expansion coefficients are expressed through recursive formulas.

For the incident field, we restrict ourselves to the case in which the incident field is the fundamental TE mode of a multilayer waveguide with no confinement of the core in one of the transverse directions. In this case, the only nonzero electric field component of the incident field is $E_{y}$, given in each individual waveguide layer as

$$
E^{y, \text { inc }}=A_{i} \exp \left(i k_{i} x-i \beta z\right)+B_{i} \exp \left(-i k_{i} x-i \beta z\right) .
$$

Here the wave number, $k_{i}$, is given by

$$
k_{i}^{2}+\beta^{2}=n_{i}^{2} k_{0}^{2}
$$

where $k_{0}$ represents the free-space wave number and $\beta$ is the propagation constant of the fundamental TE mode as determined by the layer thicknesses and the refractive indices. ${ }^{6} \quad A_{i}$ and $B_{i}$ in Eq. (4) are constants, which are given once the propagation constant is calculated. The magnetic field components of the incident field are found through Eq. (1). We denote the air above the waveguide structure layer 0; and the top cladding layer, layer 1 . We note that to ensure boundedness of the incident field it is required that $A_{0}=0$, so the incident field is described by

$$
\begin{aligned}
& E^{y, \text { inc }, 0}=B_{0} \exp \left(i k_{0} x-i \beta z\right), \\
& E^{y, \text { inc }, 1}=A_{1} \exp \left(-i k_{1} x-i \beta z\right)+B_{1} \exp \left(i k_{1} x-i \beta z\right) .
\end{aligned}
$$

The first step in deriving the recursive formulas for the Taylor expansion coefficients and thus the diffracted solution is to specify the boundary conditions on the dielectric interface that will subsequently undergo deformations. The boundary conditions for the diffracted fields $\mathbf{E}^{ \pm}$are

$$
\begin{aligned}
& n_{\delta} \times\left(\mathbf{E}^{+}-\mathbf{E}^{-}\right) \\
& =n_{\delta} \times \hat{y}\left(E^{y, \text { inc, } 1}-E^{y, \text { inc,0 }}\right) \\
& =-\left(n_{\delta} \times \hat{y}\right)\left[A_{1} \exp \left(-i k_{1} x-i \beta z\right)+B_{1}\right. \\
& \left.\times \exp \left(i k_{1} x-i \beta z\right)-B_{0} \exp \left(i k_{0} x-i \beta z\right)\right] n_{\delta} \\
& \times\left(\nabla \times \mathbf{E}^{+}-\nabla \times \mathbf{E}^{-}\right) \\
& =n_{\delta} \times\left(\nabla \times \hat{y} E^{y, \text { inc, } 1}-\nabla \times \hat{y} E^{y, \text { inc, } 0}\right) n_{\delta} \\
& \times\left\{\frac { \beta } { \omega \mu _ { 0 } } \hat { x } \left[A_{1} \exp \left(-i k_{1} x-i \beta z\right)+B_{1}\right.\right. \\
& \left.\times \exp \left(i k_{1} x-i \beta z\right)-B_{0} \exp \left(i k_{0} x-i \beta z\right)\right] \\
& +\hat{z} \frac{1}{i \omega \mu_{0}}\left[-i k_{1} A_{1} \exp \left(-i k_{1} x-i \beta z\right)+i k_{1} B_{1}\right. \\
& \left.\left.\times \exp \left(i k_{1} x-i \beta z\right)-i k_{0} B_{0} \exp \left(i k_{0} x-i \beta z\right)\right]\right\}
\end{aligned}
$$

on $x=\delta f(z, y) . \quad n_{\delta}$ is the surface normal. Given that

$$
n_{\delta}=\frac{1}{l}\left(1,-\delta f_{y},-\delta f_{z}\right),
$$

where $l$ is the length of the normal and $f_{y}$ and $f_{z}$ are the partial derivatives of $f$ with respect to $y$ and $z$, respectively. Equations (5) yield six equations, although only four of them are independent. A particular set of these is

$$
E^{3+}-E^{3-}+\delta f_{z}\left(E^{1+}-E^{1-}\right)=0
$$$$
E^{2+}-E^{2-}+\delta f_{y}\left(E^{1+}-E^{1-}\right)
$$

$$
\begin{aligned}
= & A_{1} \exp \left(-i k_{1} x-i \beta z\right)+B_{1} \exp \left(i k_{1} x-i \beta z\right) \\
& -B_{0} \exp \left(i k_{0} x-i \beta z\right)
\end{aligned}
$$




$$
\begin{aligned}
& E_{x}^{2+}-E_{x}^{2-}-\left(E_{y}^{1+}-E_{y}^{1-}\right) \\
& +\delta f_{z}\left(E_{y}^{3+}-E_{y}^{3-}-E_{z}^{2-}+E_{z}^{2-}\right) \\
& =-i k_{1} A_{1} \exp \left(-i k_{1} x-i \beta z\right)+i k_{1} B_{1} \\
& \quad \times \exp \left(i k_{1} x-i \beta z\right)-i k_{0} B_{0} \exp \left(i k_{0} x-i \beta z\right) \\
& \quad+\delta f_{z} i \beta\left[A_{1} \exp \left(-i k_{1} x-i \beta z\right)+B_{1}\right. \\
& \left.\quad \times \exp \left(i k_{1} x-i \beta z\right)-B_{0} \exp \left(i k_{0} x-i \beta z\right)\right],
\end{aligned}
$$

$$
\begin{aligned}
& E_{x}^{3+}-E_{x}^{3-}-\left(E_{z}^{1+}-E_{z}^{1-}\right) \\
& +\delta f_{y}\left[E_{y}^{3+}-E_{y}^{3-}-\left(E_{z}^{2+}-E_{z}^{2-}\right)\right] \\
& \quad=i \beta \delta f_{y}\left[A_{1} \exp \left(-i k_{1} x-i \beta z\right)\right. \\
& \left.\quad+B_{1} \exp \left(i k_{1} x-i \beta z\right)-B_{0} \exp \left(i k_{0} x-i \beta z\right)\right]
\end{aligned}
$$

Again, superscripts 1,2 , and 3 refer to $x, y$, and $z$ components, respectively.

We note that, for $\pm x> \pm \delta f(z, y)$, we also have that

$$
\nabla \cdot \mathbf{E}^{ \pm}=0
$$

or, specifically,

$$
\begin{aligned}
& E_{x}^{1+}+E_{y}^{2+}+E_{z}^{3+}=0, \\
& E_{x}^{1-}+E_{y}^{2-}+E_{z}^{3-}=0 .
\end{aligned}
$$

Subscript $x$ means a partial derivative with respect to $x$, and so on. When these divergence conditions are combined with the four transmission conditions, Eq. (6) yields six equations for the six unknown diffracted E-field components, $\mathbf{E}^{ \pm}$, at the diffracting boundary.

To recover expressions for the derivatives of the Rayleigh expansion coefficients we differentiate Eqs. (6) and (7) with respect to $\delta$ and evaluate them for $\delta=0$ and $x$ $=0$. The results for the second and third equations of Eq. (6) are

$$
\begin{aligned}
\frac{1}{n !} \frac{\partial^{n}\left(E^{2+}-E^{2-}\right)}{\partial \delta^{n}} & \sum_{p=0}^{n-1} \frac{f^{n-p}}{(n-p) !} \frac{\partial^{n-p}}{\partial x^{n-p}}\left[\frac{1}{p !} \frac{\partial^{p}\left(E^{2+}-E^{2-}\right)}{\partial \delta^{p}}\right] \\
= & -f_{y} \sum_{p=0}^{n-1} \frac{f^{n-p-1}}{(n-1-p) !} \frac{\partial^{n-1-p}}{\partial x^{n-1-p}} \\
& \times\left[\frac{1}{p !} \frac{\partial^{p}\left(E^{1+}-E^{1-}\right)}{\partial \delta^{p}}\right]+\left[A_{1}\left(-i k_{1}\right)^{n}\right. \\
& \left.+B_{1}\left(i k_{1}\right)^{n}-B_{0}\left(i k_{0}\right)^{n}\right] \frac{f^{n}}{n !} \exp (-i \beta z),
\end{aligned}
$$

$$
\begin{aligned}
\frac{1}{n !} \frac{\partial^{n}}{\partial \delta^{n}} & {\left[E_{x}^{2+}-E_{x}^{2-}-\left(E_{y}^{1+}-E_{y}^{1-}\right)\right] } \\
= & -\sum_{p=0}^{n-1} \frac{f^{n-p}}{(n-p) !} \frac{\partial^{n-p}}{\partial x^{n-p}} \\
& \times\left\{\frac{1}{p !} \frac{\partial^{p}\left[E_{x}^{2+}-E_{x}^{2-}-\left(E_{y}^{1+}-E_{y}^{1-}\right)\right]}{\partial \delta^{p}}\right\} \\
& -f_{z} \sum_{p=0}^{n-1} \frac{f^{n-p-1}}{(n-1-p) !} \frac{\partial^{n-1-p}}{\partial x^{n-1-p}} \\
& \times\left[\frac{1}{p !} \frac{\partial^{p}\left(E_{y}^{3+}-E_{y}^{3-}-E_{z}^{2+}+E_{z}^{2-}\right)}{\partial \delta^{p}}\right] \\
& +\left[-A_{1}\left(-i k_{1}\right)^{n+1}+B_{1}\left(i k_{1}\right)^{n+1}\right. \\
& \left.+B_{0}\left(i k_{0}\right)^{n+1}\right] \frac{f^{n}}{n !} \exp ^{2-i \beta z)+\beta f_{y}\left[A_{1}\left(-i k_{1}\right)^{n-1}\right.} \\
& \left.+B_{1}\left(i k_{1}\right)^{n-1}-B_{0}\left(i k_{0}\right)^{n-1}\right] \frac{f^{n-1}}{(n-1) !} \exp (-i \beta z)
\end{aligned}
$$

Introducing the Fourier coefficients of $f^{l} / l$ as

$$
\frac{f(y, z)^{l}}{l !}=\sum_{t=-l F_{y}}^{l F_{y}} \sum_{q=-l F_{z}}^{l F_{z}} C_{l,(t, q)} \exp \left[i\left(K_{y} t y+K_{z} q z\right)\right]
$$

we note that

$$
\begin{aligned}
f_{z} \frac{f^{l-1}}{(l-1) !}= & \sum_{-l F<t, q<l F} C_{l,(t, q)}\left(i K_{z} q z\right) \\
& \times \exp \left[i\left(K_{y} t y+K_{z} q z\right)\right], \\
f_{y} \frac{f^{l-1}}{(l-1) !}= & \sum_{-l F<t, q<l F} C_{l,(t, q)}\left(i K_{y} t y\right) \\
& \times \exp \left[i\left(K_{y} t y+K_{z} q z\right)\right] .
\end{aligned}
$$

It follows from Eqs. (2) and (3) that

$$
\begin{aligned}
\frac{1}{p !} \frac{\partial^{p} \mathbf{E}^{ \pm}}{\partial \delta^{p}}(x, y, z ; \delta=0) \\
=\sum_{r, s} \frac{1}{p !} \frac{\partial^{p} \mathbf{B}_{r, s}^{ \pm}}{\partial \delta^{p}}(0) \exp \left( \pm i \alpha_{r, s}^{ \pm} x+i \gamma_{s} y-i \beta_{r} z\right) \\
=\sum_{r, s} \mathbf{d}_{p,(r, s)}^{ \pm} \exp \left( \pm i \alpha_{r, s}^{ \pm} x+i \gamma_{s} y-i \beta_{r} z\right),
\end{aligned}
$$

and, substituting this equation into Eqs. (8) along with the Fourier expansions, we get 


$$
\begin{aligned}
& \sum_{r, s}\left[d_{p,(r, s)}^{2+}-d_{p,(r, s)}^{2-}\right] \exp \left[i\left(\gamma_{s} y+\beta_{r} z\right)\right] \\
& =-\sum_{p=0}^{n-1}\left\{\sum_{-(n-p) F<t, q<(n-p) F} C_{n-p,(t, q)} \exp \left[i\left(K_{y} t y+K_{z} q z\right)\right]\right\}\left\{\sum_{l, m}\left[\left(i \alpha_{l, m}^{+}\right)^{n-p} d_{p,(l, m)}^{2+}-\left(-i \alpha_{l, m}^{-}\right)^{n-p} d_{p,(l, m)}^{2-}\right]\right. \\
& \left.\times \exp \left[i\left(\gamma_{s} y+\beta_{r} z\right)\right]\right\}-\sum_{p=0}^{n-1}\left\{\sum_{-(n-p) F<t, q<(n-p) F} C_{n-p,(t, q)}\left(i K_{y} t\right) \exp \left[i\left(K_{y} t y+K_{z} q z\right)\right]\right\} \\
& \times\left\{\sum_{l, m}\left[\left(i \alpha_{l, m}^{+}\right)^{n-p-1} d_{p,(l, m)}^{1+}-\left(-i \alpha_{l, m}^{-}\right)^{n-p-1} d_{p,(l, m)}^{1-}\right] \exp \left[i\left(\gamma_{s} y+\beta_{r} z\right)\right]\right\} \\
& +\sum_{p=0}^{n-1}\left\{\sum_{-(n-p) F<t, q<(n-p) F} C_{n-p,(t, q)} \exp \left[i\left(K_{y} t y+K_{z} q z\right)\right]\right\}\left[A_{1}\left(-i k_{1}\right)^{n}+B_{1}\left(i k_{1}\right)^{n}-B_{0}\left(i k_{0}\right)^{n}\right] \exp (-i \beta z) \text {, } \\
& \sum_{r, s}\left\{i \alpha_{r, s}^{+} d_{p,(r, s)}^{2+}+i \alpha_{r, s}^{-} d_{p,(r, s)}^{2-}-\left[i \gamma_{s} d_{p,(r, s)}^{1+}-i \gamma_{s} d_{p,(r, s)}^{1-}\right]\right\} \exp \left[i\left(\gamma_{s} y+\beta_{r} z\right)\right] \\
& =-\sum_{p=0}^{n-1}\left\{\sum_{-(n-p) F<t, q<(n-p) F} C_{n-p,(t, q)} \exp \left[i\left(K_{y} t y+K_{z} q z\right)\right]\right\} \\
& \times\left\{\sum_{l, m}\left[\left(i \alpha_{l, m}^{+}\right)^{n-p+1} d_{p,(l, m)}^{2+}-\left(-i \alpha_{l, m}^{-}\right)^{n-p+1} d_{p,(l, m)}^{2-}-\left(i \alpha_{l, m}^{+}\right)^{n-p}\left(i \gamma_{s}\right) d_{p,(l, m)}^{1+}+\left(i \alpha_{l, m}^{-}\right)^{n-p}\left(i \gamma_{s}\right) d_{p,(l, m)}^{1-}\right]\right. \\
& \left.\times \exp \left[i\left(\gamma_{s} y+\beta_{r} z\right)\right]\right\}-\sum_{p=0}^{n-1}\left\{\sum_{-(n-p) F<t, q<(n-p) F} C_{n-p,(t, q)}\left(i K_{z} q\right) \exp \left[i\left(K_{y} t y+K_{z} q z\right)\right]\right\} \\
& \times\left\{\sum _ { l , m } \left(( i \alpha _ { l , m } ^ { + } ) ^ { n - p - 1 } \left[i \gamma_{s} d_{p,(l, m)}^{3+}-\left(-i \alpha_{l, m}^{-}\right)^{n-p-1} i \gamma_{s} d_{p,(l, m)}^{3-}-\left(i \alpha_{l, m}^{+}\right)^{n-p-1}\left(i \beta_{r}\right) d_{p,(l, m)}^{2+}\right.\right.\right. \\
& \left.\left.+\left(i \alpha_{l, m}^{-}\right)^{n-p-1}\left(i \beta_{r}\right) d_{p,(l, m)}^{2-}\right] \exp \left[i\left(\gamma_{s} y+\beta_{r} z\right)\right]\right\}+\sum_{p=0}^{n-1}\left\{\sum_{-(n-p) F<t, q<(n-p) F} C_{n-p,(t, q)} \exp \left[i\left(K_{y} t y+K_{z} q z\right)\right]\right\} \\
& \times\left[-A_{1}\left(-i k_{1}\right)^{n+1}+B_{1}\left(i k_{1}\right)^{n+1}+B_{0}\left(i k_{0}\right)^{n+1}\right] \exp (-i \beta z)+\beta \sum_{p=0}^{n-1}\left\{\sum_{-(n-p) F<t, q<(n-p) F} C_{n-p,(t, q)}\right. \\
& \left.\times\left(i K_{y} t\right) \exp \left[i\left(K_{y} t y+K_{z} q z\right)\right]\right\}\left[A_{1}\left(-i k_{1}\right)^{n-1}+B_{1}\left(i k_{1}\right)^{n-1}-B_{0}\left(i k_{0}\right)^{n-1}\right] \exp (-i \beta z) .
\end{aligned}
$$

We next write the Fourier series expansions for the right-hand sides of Eqs. (9) and (10); the first one is

$$
\begin{aligned}
& -\sum_{p=0}^{n-1} \sum_{l, m} \sum_{-(n-p) F<t, q<(n-p) F}\left[\left(i \alpha_{l, m}^{+}\right)^{n-p} d_{p,(l, m)}^{2+}-\left(-i \alpha_{l, m}^{-}\right)^{n-p} d_{p,(l, m)}^{2-}\right] C_{n-p,(t, q)} \exp \left[i\left(K_{y} t y+K_{z} q z\right)\right] \exp \left[i\left(\gamma_{s} y+\beta_{r} z\right)\right] \\
& \quad-\sum_{p=0}^{n-1} \sum_{-(n-p) F<t, q<(n-p) F} \sum_{l, m}\left[\left(i \alpha_{l, m}^{+}\right)^{n-p-1} d_{p,(l, m)}^{1+}-\left(-i \alpha_{l, m}^{-}\right)^{n-p-1} d_{p,(l, m)}^{1-}\right]\left(i K_{y} t\right) C_{n-p,(t, q)} \exp \left[i \left(K_{y} t y\right.\right. \\
& \left.\left.\quad+K_{z} q z\right)\right] \exp \left[i\left(\gamma_{s} y+\beta_{r} z\right)\right]+\sum_{-(n-p) F<t, q<(n-p) F}\left[A_{1}\left(-i k_{1}\right)^{n}+B_{1}\left(i k_{1}\right)^{n}-B_{0}\left(i k_{0}\right)^{n}\right] C_{p,(t, q)} \exp \left[i\left(\gamma_{s} y+\beta_{r} z\right)\right],
\end{aligned}
$$

and that for Eq. (10) is 


$$
\begin{aligned}
& -\sum_{p=0}^{n-1} \sum_{l, m} \sum_{-(n-p) F<t, q<(n-p) F}\left[\left(i \alpha_{l, m}^{+}\right)^{n-p+1} d_{p,(l, m)}^{2+}-\left(-i \alpha_{l, m}^{-}\right)^{n-p+1} d_{p,(l, m)}^{2-}-\left(i \alpha_{l, m}^{+}\right)^{n-p}\left(i \gamma_{s}\right) d_{p,(l, m)}^{1+}\right. \\
& \left.\quad+\left(i \alpha_{l, m}^{-}\right)^{n-p}\left(i \gamma_{s}\right) d_{p,(l, m)}^{1-}\right] C_{n-p,(t, q)} \exp \left[i\left(K_{y} t y+K_{z} q z\right)\right] \exp \left[i\left(\gamma_{s} y+\beta_{r} z\right)\right]-\sum_{p=0}^{n-1} \sum_{l, m} \sum_{-(n-p) F<t, q<(n-p) F} \\
& \quad \times\left[\left(i \alpha_{l, m}^{+}\right)^{n-p-1}\left(i \gamma_{s}\right) d_{p,(l, m)}^{3+}-\left(-i \alpha_{l, m}^{-}\right)^{n-p-1} i \gamma_{s} d_{p,(l, m)}^{3-}-\left(i \alpha_{l, m}^{+}\right)^{n-p-1}\left(i \beta_{r}\right) d_{p,(l, m)}^{2+}+\left(i \alpha_{l, m}^{-}\right)^{n-p-1}\left(i \beta_{r}\right) d_{p,(l, m)}^{2-}\right] \\
& \quad \times\left(i K_{z} q\right) C_{n-p,(t, q)} \exp \left[i\left(K_{y} t y+K_{z} q z\right)\right] \exp \left[i\left(\gamma_{s} y+\beta_{r} z\right)\right]+\sum_{p=0}^{n-1} \sum_{-(n-p) F<t, q<(n-p) F} \\
& \quad \times\left\{-A_{1}\left(-i k_{1}\right)^{n+1}+B_{1}\left(i k_{1}\right)^{n+1}+B_{0}\left(i k_{0}\right)^{n+1}+\beta\left[A_{1}\left(-i k_{1}\right)^{n-1}+B_{1}\left(i k_{1}\right)^{n-1}-B_{0}\left(i k_{0}\right)^{n-1}\right]\right\} \\
& \quad \times C_{n,(t, q)} \exp \left[i\left(\gamma_{s} y+\beta_{r} z\right)\right] .
\end{aligned}
$$

Given that

$$
\exp \left[i\left(K_{y} t y+K_{z} q z\right)\right] \exp \left[i\left(\gamma_{s} y+\beta_{r} z\right)\right]=\exp \left[i\left(\gamma_{s+t} y+\beta_{r+q} z\right)\right],
$$

we can replace $t$ by $s-l$ and $q$ by $r-m$ in the inner sum. Then, finally, by equating Fourier coefficients, we arrive at the recursive formulas

$$
\begin{aligned}
& d_{n,(r, s)}^{2+}-d_{n,(r, s)}^{2-} \\
& =\left[-B_{0}\left(i k_{0}\right)^{n}+A_{1}\left(-i k_{1}\right)^{n}+B_{1}\left(i k_{1}\right)^{n}\right] C_{n,(r, s)}-\sum_{p=0}^{n-1} \sum_{l=\max [-p F, r-(n-p) F]}^{\min [p F, r+(n-p) F]} \sum_{m=\max [-p F, s-(n-p) F]}^{\min [p F, s+(n-p) F]}\left\{\left(i \alpha_{l, m}^{+}\right)^{n-p} d_{p,(l, m)}^{2+}\right. \\
& \left.-\left(-i \alpha_{l, m}^{-}\right)^{n-p} d_{p,(l, m)}^{2-}+\left[\left(i \alpha_{l, m}^{+}\right)^{n-1-p} d_{p,(l, m)}^{1+}-\left(-i \alpha_{l, m}^{-}\right)^{n-1-p} d_{p,(l, m)}^{1-}\right]\left[i K_{y}(s-m)\right]\right\} C_{n-p,(r-t, s-m)}, \\
& \alpha_{r, s}^{+} d_{n,(r, s)}^{2+}+\alpha_{r, s}^{-} d_{n,(r, s)}^{2-}-\gamma_{s} d_{n,(r, s)}^{1+}+\gamma_{s} d_{n,(r, s)}^{1-} \\
& =\left\{-A_{1}\left(-i k_{1}\right)^{n+1}+B_{1}\left(i k_{1}\right)^{n+1}+B_{0}\left(i k_{0}\right)^{n+1}+i \beta\left[A_{1}\left(-i k_{1}\right)^{n-1}+B_{1}\left(i k_{1}\right)^{n-1}-B_{0}\left(i k_{0}\right)^{n-1}\right]\left(i K_{z} r\right)\right\} C_{n,(r, s)} \\
& -\sum_{p=0}^{n-1} \sum_{l=\max [-p F, r-(n-p) F]}^{\min [p F, r+(n-p) F]} \sum_{m=\max [-p F, s-(n-p) F]}^{\min [p F, s+(n-p) F]}\left\{\alpha_{l, m}^{+}\left(i \alpha_{l, m}^{+}\right)^{n-p} d_{p,(r, s)}^{2+}+\alpha_{l, m}^{-}\left(-i \alpha_{l, m}^{-}\right)^{n-p} d_{p,(r, s)}^{2-}-\gamma_{s}\left(i \alpha_{l, m}^{+}\right)^{n-p} d_{p,(r, s)}^{1+}\right. \\
& +\gamma_{s}\left(-i \alpha_{l, m}^{-}\right)^{n-p} d_{p,(r, s)}^{1-}+\left[\gamma_{s}\left(i \alpha_{l, m}^{+}\right)^{n-p-1} d_{p,(r, s)}^{3+}-\gamma_{s}\left(-i \alpha_{l, m}^{-}\right)^{n-p-1} d_{p,(r, s)}^{3-} \beta_{r}\left(i \alpha_{l, m}^{+}\right)^{n-p-1} d_{p,(r, s)}^{2+}\right. \\
& \left.\left.+\beta_{r}\left(-i \alpha_{l, m}^{-}\right)^{n-p-1} d_{p,(r, s)}^{2-}\right]\left[i K_{z}(r-l)\right]\right\} C_{n-p,(r-l, s-m)},
\end{aligned}
$$

where we have exploited the fact that

$$
d_{p,(l, m)}^{i, \pm}=0, \quad|l|>p F, \quad|m|>p F .
$$

Similar calculations for the first and fourth equations of Eqs. (6) lead one to obtain the recursive formulas

$$
\begin{aligned}
& d_{n,(r, s)}^{3+}-d_{n,(r, s)}^{3-}=-\sum_{p=0}^{n-1} \sum_{l=\max [-p F, r-(n-p) F]}^{\min [p F, r+(n-p) F]} \sum_{m=\max [-p F, s-(n-p) F]}^{\min [p F, s+(n-p) F]}\left\{\left(i \alpha_{l, m}^{+}\right)^{n-p} d_{p,(r, s)}^{3+}-\left(-i \alpha_{l, m}^{-}\right)^{n-p} d_{p,(r, s)}^{3-}\right. \\
& \left.\quad+\left[\left(i \alpha_{l, m}^{+}\right)^{n-1-p} d_{p,(l, m)}^{1+}-\left(-i \alpha_{l, m}^{-}\right)^{n-1-p} d_{p,(l, m)}^{1-}\right]\left[i K_{z}(r-l)\right]\right\} C_{n-p,(r-l, s-m)}, \\
& \alpha_{r, s}^{+} d_{n,(r, s)}^{3+}+\alpha_{r, s}^{-} d_{n,(r, s)}^{3-}-\beta_{r} d_{n,(r, s)}^{1+}+\beta_{r} d_{n,(r, s)}^{1-} \\
& =\left[A_{1}\left(-i k_{1}\right)^{n-1}+B_{1}\left(i k_{1}\right)^{n-1}-B_{0}\left(i k_{0}\right)^{n-1}\right] i \beta\left(i K_{1} r\right) C_{n,(r, s)} \\
& \quad-\sum_{p=0}^{n-1} \min [p F, r+(n-p) F] \\
& \quad \sum_{l=\max [-p F, r-(n-p) F]} \min [p F, s+(n-p) F] \\
& \quad-\alpha_{l, m}^{-}\left(-i \alpha_{l, m}^{-}\right)^{n-p} d_{p,(r, s)}^{3-}+\left[\gamma_{s}\left(i \alpha_{l, m}^{+}\right)^{n-1-p} d_{p,(l, m)}^{3+}-\gamma_{s}\left(-i \alpha_{l, m}^{-}\right)^{n-1-p} d_{p,(l, m)}^{3-}-\beta_{r}\left(i \alpha_{l, m}^{+}\right)^{n-1-p} d_{p,(l, m)}^{2+}\right.
\end{aligned}
$$

These equations, in combination with the equivalence to Eq. (7), 


$$
\begin{array}{r}
\alpha_{r, s}^{+} d_{n,(r, s)}^{1+}+\gamma_{s} d_{n,(r, s)}^{2+}-\beta_{r} d_{n,(r, s)}^{3+}=0, \\
-\alpha_{r, s}^{-} d_{n,(r, s)}^{1-}+\gamma_{s} d_{n,(r, s)}^{2-}-\beta_{r} d_{n,(r, s)}^{3-}=0,
\end{array}
$$

give a set of equations from which one can compute recursively the Rayleigh power series expansion coefficients and hence the Rayleigh expansion of the radiation fields. The radius of convergence of the power series expansion is generally rather small. To enhance the radius of convergence we recast the Taylor expansion as a Padé approximation. ${ }^{7}$ We generally find that use of an $[M, M]$ approximant, i.e., of the same order of the polynomial in the numerator and the denominator of the rational polynomial, yields the fastest convergence. As for the computation of the Fourier spectrum of the surface relief, we use the fast Fourier transform for enhanced computational speed.

\section{FIELD COMPUTATIONS}

In principle, the radiated fields can be computed anywhere outside $|x|>\delta f(z, y)$ by use of the Rayleigh expansion [Eq. (2)]. However, because of the inherent periodicity introduced by the Rayleigh expansion itself, this procedure can lead to problems with waves leaving the computational domain at one boundary in the $z-y$-plane and reentering at the opposite boundary. To avoid such problems, we exploit near- and far-field integration techniques based on the surface-equivalence theorem $^{8}$ by which the fields anywhere above an aperture covering the grating surface can be computed rigorously from equivalent sources on the aperture. To maintain high accuracy, we compute the diffracted field on a set of quadrature points on which high-order integration can be performed, thus ensuring that the high accuracy is maintained everywhere.

\section{NUMERICAL VERIFICATION}

To verify the numerical scheme derived in Section 4 , we compare its results with the numerical results obtained from a spectral collocation scheme, computing direct time-domain solutions to Maxwell's equations rigorously and with spectral accuracy. ${ }^{2}$

For the purpose of verification we use the generic surface-relief grating given as

$$
\begin{aligned}
f_{\delta}(z, y)= & A \exp \left[-\left(\frac{z-z_{0}}{w_{z}}\right)^{2}\right]\left[-\left(\frac{y-y_{0}}{w_{y}}\right)^{2}\right] \\
& \times \cos \left(2 \pi\left\{a_{0}+a_{1}\left[r(z, y)-r_{0}\right]\right\}\right. \\
& \left.\times\left[r(z, y)-r_{0}\right]\right) .
\end{aligned}
$$

Here $A$ is the amplitude of the relief, $w_{z}$ and $w_{y}$ represent the widths of the Gaussian tapering of the relief, $\left(z_{0}, y_{0}\right)$ is the center of the relief, and $a_{0}=1 / \Lambda$ for the unchirped relief, where $a_{1}$ is the chirp parameter and $r(z, y)$ is the radial variable, given by

$$
r(z, y)=\left[\left(z-z_{c}\right)^{2}+\left(y-y_{c}\right)^{2}\right]^{1 / 2} .
$$

Here $\left(z_{c}, y_{c}\right)$ is the center of the concentric circles that describe the grating with $r_{0}=r\left(z_{0}, y_{0}\right)$.
In what follows, we utilize the guidelines for the domain size and the number of Fourier modes that are necessary to simulate a grating coupler of finite extent reached during our previous development of the twodimensional boundary variation method for modeling grating couplers. ${ }^{3}$ As a rule of thumb we found that using a domain length four to five times the Gaussian width, $w$, suffices to simulate a finite coupler, whereas using three or more Fourier modes per grating period accurately resolves the surface relief. All dimensions in the examples that follow are normalized with the free-space wavelength $\lambda$ of the incident field.

As a first example, we examine the far-field radiation from a surface relief described by Eq. (14) with the following parameters: $A=0.1, \quad w_{z}=w_{y}=4, \quad\left(z_{0}, y_{0}\right)$ $=(0,0), \quad a_{0}=1 / 1.4213, \quad a_{1}=0.01, \quad$ and $\quad\left(z_{c}, y_{c}\right)$ $=(0,-155)$. In Fig. 2 we display two line scans of the far-field intensity for $\phi=0$ and $\theta=\pi / 2$, respectively. Evidently, we have excellent agreement between the two methods, as we find the results to be nearly indistinguishable lines over 4 orders of magnitude. As expected, we also find that the particular relief yields a perpendicular output angle $(\theta=\pi / 2)$ for the -1 st diffraction order.

Let us also evaluate the quality of the radiated fields in the near field in a plane just above the surface relief. The result of this direct comparison is shown in Fig. 3, which, again, confirms the excellent agreement between the two methods also in the near field.

To gain an impression of the memory and computational time requirements for the two methods, in Table 1

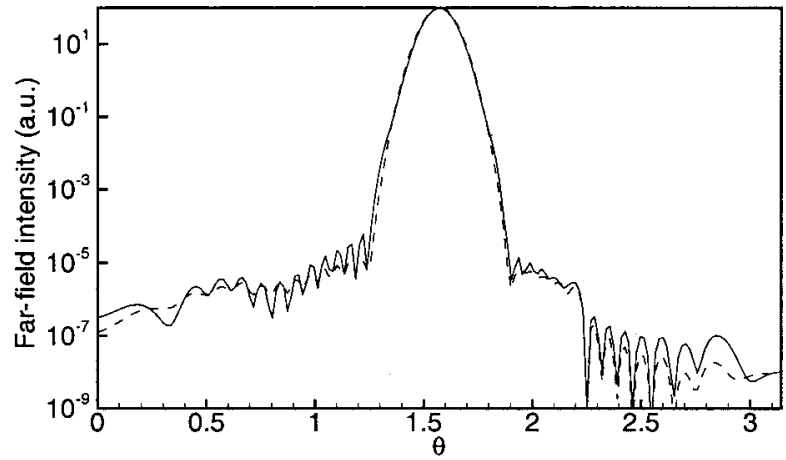

(a)

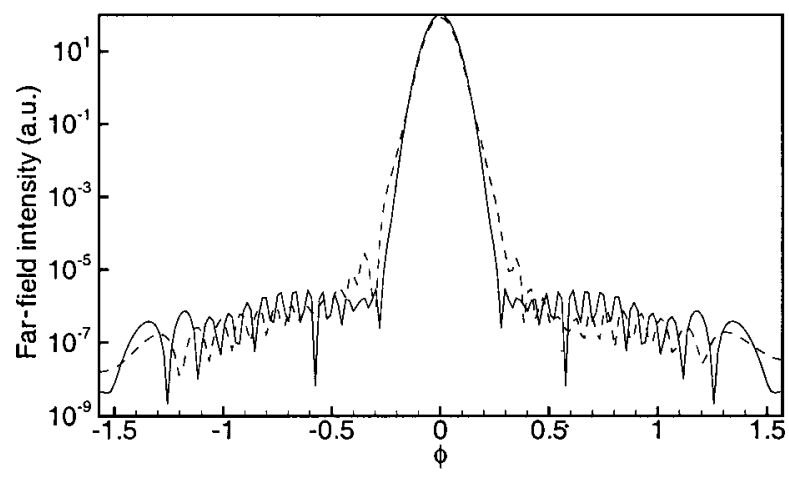

(b)

Fig. 2. Line scans of far-field radiation from a surface-relief grating along (a) $\phi=0$ and (b) $\theta=\pi / 2$, comparing the proposed boundary variation method (solid curves) and a rigorous spectral collocation method (dashed curves). 


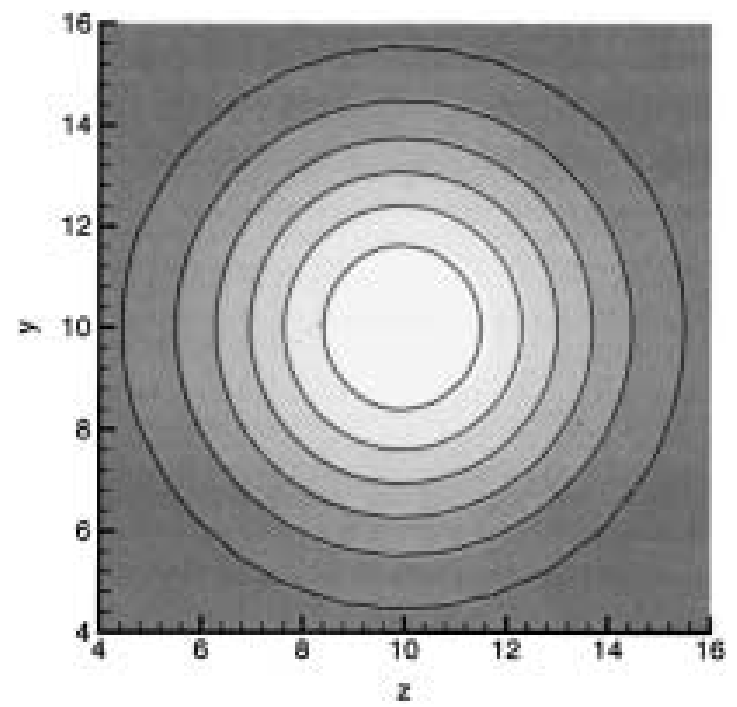

Fig. 3. Contour plot of the field variable, $E_{y}$, in an aperture plane one wavelength above the surface relief, computed by the spectral collocation method (solid gray-scale contours) and the boundary variation method (line contours).

Table 1. Comparison of Memory Usage and Computational Time for the Boundary Variation Method and the Spectral Collocation Method for Three-Dimensional Surface-Relief Scattering Problems

\begin{tabular}{ccc}
\hline Method $^{a}$ & Memory (Mbytes) & CPU Time (min) \\
\hline BV & 191 & 38 \\
SC & 3568 & 14,100 \\
\hline
\end{tabular}

${ }^{a} \mathrm{BV}$, boundary variation; SC, spectral collocation code.

we compare these numbers for the example discussed above. Both codes were run on an IBM RS/6000 SP parallel computer equipped with Power 3 CPUs. The boundary variation method was run on a single CPU, whereas the spectral collocation code was run in parallel on 16 processors. For direct comparison, the numbers for the spectral collocation code in Table 1 represent the estimated numbers, if the code were run on a single processor.

From the table it is evident that a significant reduction in both memory and CPU time is possible from use of the boundary variation method. A reduction by a factor of $\sim 19$ in the memory requirements is possible, and the CPU time consumption is a factor of 371 lower for the boundary variation method than for the spectral collocation code.

\section{ANALYSIS OF FOCUSING WAVEGUIDE GRATING COUPLERS}

Having verified the validity of the boundary variation method by careful comparisons with the direct and highly accurate spectral collocation method, we are now in a position to consider the near-field radiation from a number of nontrivial examples of grating couplers.

As a first example, we consider the near-field radiation from the same grating coupler as in Sec. 5, however with the center of the concentric circles shifted to $\left(z_{c}, y_{c}\right)$ $=(0,-35.17)$ and the chirp parameter $a_{2}=0.02$. Based on a simple geometrical optics analysis this should lead to focusing in the $y$ direction at a distance of $25 \lambda$ from the surface relief.

In Fig. 4 we show contour plots of the dominant field component, $E_{y}$, from which it is evident that the surface relief does indeed lead to focusing of the diffracted field. The focal plane, however, lies at a distance of 20 wavelengths away from the surface, rather than 25 wavelengths as suggested by the simple analysis. The shift of the focal plane toward the surface relief is consistent with previous two-dimensional results. ${ }^{2,3,9}$

To enable the focal plane to shift further away from the surface relief it is necessary to use a larger grating. In

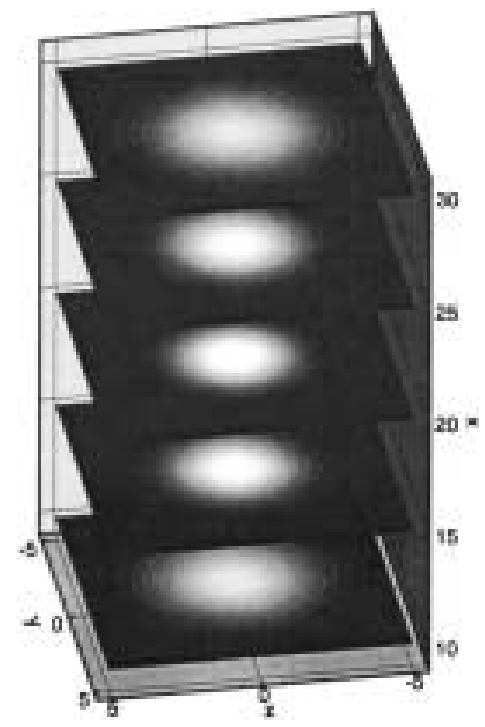

Fig. 4. Contour plots of the near-field radiation from a threedimensional grating coupler, illustrating focusing at $20 \lambda$ away from the surface relief.

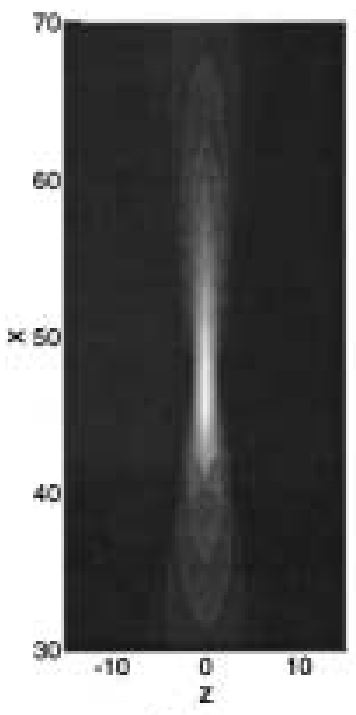

(a)

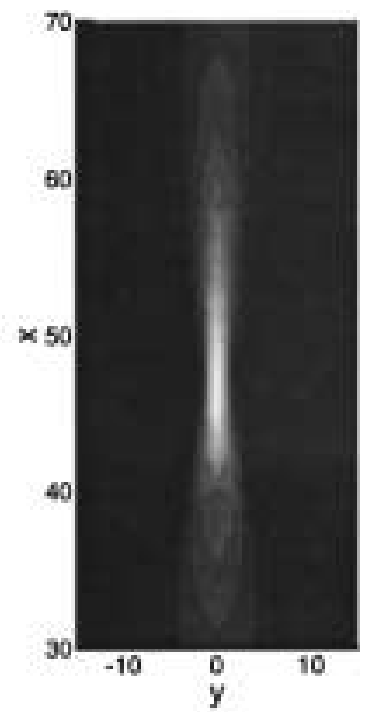

(b)
Fig. 5. Contour plots of $E_{y}$ of near-field radiation from a threedimensional grating coupler for (a) $y=0$ and (b) $z=0$. Gaussian truncation widths are $w_{z}=w_{y}=16 \lambda$. 


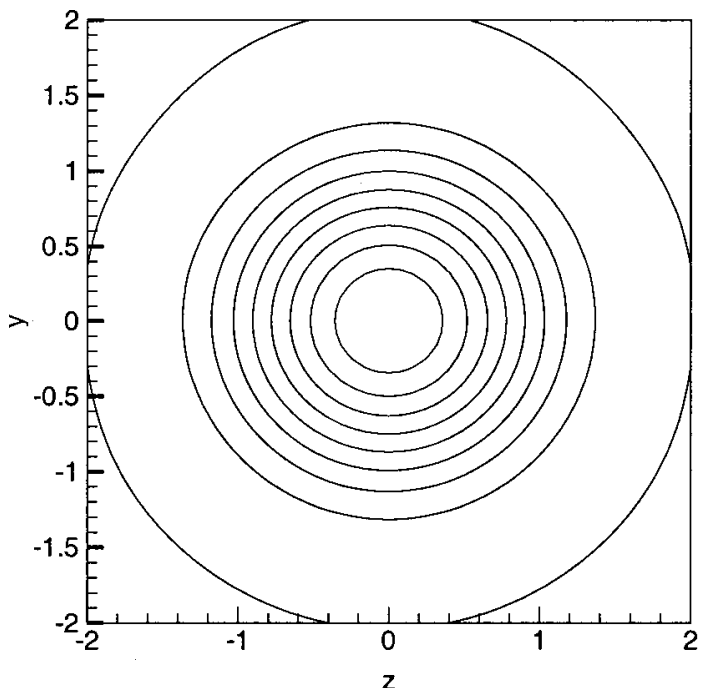

Fig. 6. Contour plot of the $E_{y}$ field component in the focal plane at $x=47$.

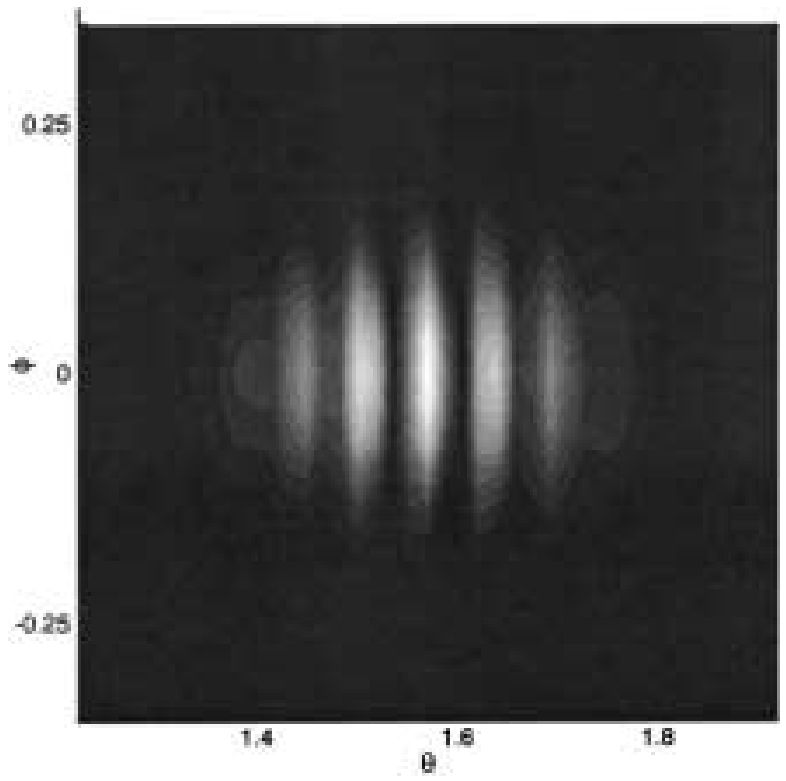

Fig. 7. Far-field radiation from a focusing grating coupler with multiplexed surface relief.

the next example, we analyze a surface relief with $w_{z}=w_{y}=16$ and a total length of the computational domain in both the $z$ and $y$ directions of 80 wavelengths. The chirp parameter is now $a_{1}=0.01$, and the center of the grating circles is located at $\left(z_{c}, y_{c}\right)=(0,-71.1)$,

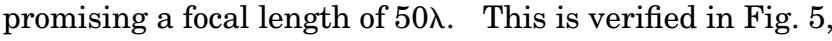
which illustrates the contour plots in two planes $(y=0$ and $z=0$ ) of the dominant field component $E_{y}$. The contour plots clearly illustrate that the fields are nearly identical in the two planes, indicating a rotational symmetry about the optical axis. This symmetry is confirmed by the contour plot in the focal plane at $x=47$ shown in Fig. 6 . This symmetry indicates a good balance between the chirp parameter and the center and radii of the circles that define the surface relief maxima, which in turn determine the focal length. As before, however, we find a focal shift toward the surface relief, although it is relatively shorter than for the smaller relief analyzed above.

As a final example we consider a focusing grating coupler with a multiplexed surface relief to permit focusing in two spots. The surface relief is now described by

$$
\begin{aligned}
f_{\delta}(z, y)= & A \exp \left[-\left(\frac{z}{w_{z}}\right)^{2}-\left(\frac{y}{w_{y}}\right)^{2}\right] \\
& \times \cos \left[K_{z}\left(r-r_{0}\right)+2 \pi a_{1}\left(r-r_{0}\right)^{2}\right] \\
& \times \cos \left[-1 / 2\left(r-r_{0}\right)+2 \pi a_{1}\left(r-r_{0}\right)^{2}\right] .
\end{aligned}
$$

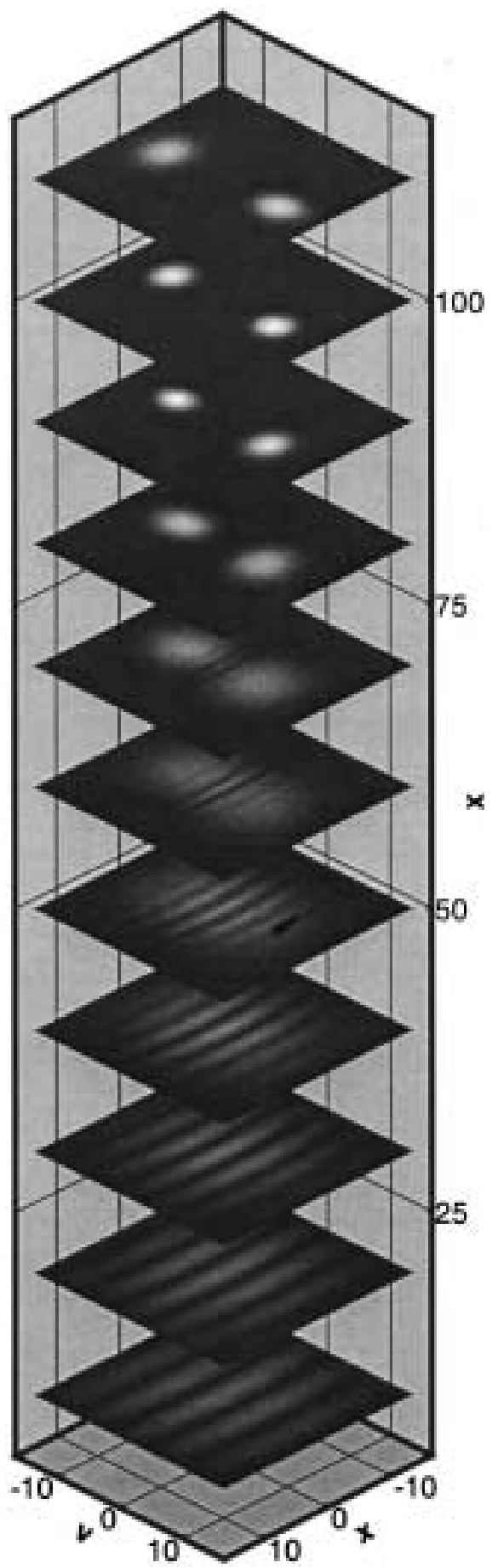

Fig. 8. Contour plots of $E_{y}$ at several distances from the surface relief for a multiplexed grating. 
The surface relief has two chirped periodic functions whose grating wave vectors differ by 1 such that the output angle between the two -1 st diffraction orders is $17.19^{\circ}$ in $\theta$. The corresponding far-field pattern is shown in Fig. 7.

The far-field radiation pattern bears evidence of the multiplexing of two gratings with different grating periods, and it is not apparent that the surface relief will produce a simultaneous focusing in two spots. To appreciate this dual focus we shall need to consider the near-field radiation from the focusing grating coupler, as illustrated in Fig. 8, which shows contour plots of $E_{y}$ at several distances from the surface relief. It is apparent that the desired dual-focus behavior of the focusing grating coupler is achieved as the radiation is focused into two spots of equal intensity at a distance of 100 wavelengths from the surface relief. This result could be expected based on the grating specification.

\section{CONCLUSIONS}

The modeling of diffractive optical elements is one of significant contemporary interest because of the possibilities that components offer as an enabling technology for the realization of miniaturized optical systems. However, the numerical modeling and design of such elements continues to challenge existing numerical techniques, and to improve current designs further will require new approaches.

In this paper we have proposed a novel boundary variation method for the analysis of full three-dimensional waveguide grating couplers with general two-dimensional finite surface reliefs. The validity and versatility of the proposed technique have been thoroughly verified by detailed comparisons with direct time-domain solutions of Maxwell's equations by use of spectral collocation methods. Besides being accurate, the proposed boundary variation method has also been shown to be dramatically faster than the direct time-domain method, suggesting that the boundary variation method is an excellent candidate for the forward solver in an optimal design process.

\section{ACKNOWLEDGMENTS}

J. S. Hesthaven gratefully acknowledges the partial support of the U.S. Air Force Office of Scientific Research/ Defense Advanced Research Projects Agency under contract F49620-1-0426 and, as a Sloan research fellow, of the Alfred P. Sloan Foundation.

P. G. Dinesen's e-mail address is palle.dinesen @risoe.dk; that of J. S. Hesthaven is jan.hesthaven @brown.edu.

\section{REFERENCES}

1. J. S. Hesthaven, P. G. Dinesen, and J.-P. Lynov, "Spectral collocation time-domain modeling of diffractive optical elements," J. Comput. Phys. 155, 287-306 (1999).

2. P. G. Dinesen and J. S. Hesthaven, "Rigorous 3-d analysis of focusing grating couplers using a spectral collocation method," in Diffractive Optics and Micro-Optics, Vol. 41 of OSA Trends in Optics and Photonics (Optical Society of America, Washington, D.C., 2000), pp. 81-83.

3. P. G. Dinesen and J. S. Hesthaven, "Fast and accurate modeling of waveguide grating couplers," J. Opt. Soc. Am. A 17, 1565-1572 (2000).

4. O. P. Bruno and F. Reitich, "Numerical solution of diffraction problems: a method of variation of boundaries," J. Opt. Soc. Am. A 10, 1168-1175 (1993).

5. O. P. Bruno and F. Reitich, "Numerical solution of diffraction problems: a method of variation of boundaries. III. Doubly periodic gratings," J. Opt. Soc. Am. A 10, 2551-2562 (1993).

6. R. Syms and J. Cozens, Optical Guided Waves and Devices (McGraw-Hill, New York, 1993), p. 114.

7. O. P. Bruno and F. Reitich, "Numerical solution of diffraction problems: a method of variation of boundaries. II. Finitely conducting gratings, Padé approximants, and singularities," J. Opt. Soc. Am. A 10, 2307-2316 (1993).

8. S. A. Schelknuoff, "Some equivalence theorems of electromagnetics and their application to radiation problems," Bell Syst. Tech. J. 15, 92-112 (1936).

9. D. W. Prather, M. S. Mirotznik, and J. N. Mait, "Boundary integral methods applied to the analysis of diffractive optical elements," J. Opt. Soc. Am. A 14, 34-43 (1997). 